\title{
The Inverse Gaussian Beam Stack Imaging Method of Cross-well Seism
}

\author{
Feilong Yang *, Zhengrong Wei, Qingchun Li \\ Chang'an University \\ College of the Geological Engineering and Geomatics \\ Xi'an, China \\ Feilongyang2006@126.com
}

\author{
Xiaobin Qu \\ Shaanxi Yanchang Petroleum Corp. Ltd \\ Oil and Gas Exploitation Company \\ Yan'an, China
}

\begin{abstract}
Cross-well seismic has the characteristic of high resolution. It can solve the exploration problems of stratigraphic trap and lithological trap in small scale and complex structure. In order to solve the problems of cross-well seismic wave imaging effectively, the paper uses Gauss beam forward method to obtain the velocity field and reflection points in complex structure; The common reflection point gathers are gotten by using the idea of inverse Gaussian beam to migrate the reflection wave field to the correct position; Then the imaging field characteristics can be acquired through stacking data in an appropriate bin size. A large number of model tests show that, the inverse Gaussian beam stack imaging method of cross-well seismic can effectively inverse the underground structure. The method is used in the cross-well seismic imaging of $K$ area, the results show that this method could accurately and rapidly image the underground complex and small scale structure characteristics, and provide the basis for further seismic data processing.
\end{abstract}

Keywords-Cross-well, Gaussian beam forward, stack imaging, inverse Gaussian beam

\section{INTRODUCTION}

With the development of the oil, gas and mineral resources exploration, the targets of exploration mainly fasten on stratigraphic and lithological trap with small scale, complex structure, and physical property changed severely, so we need a new geophysical exploration method to improve the lateral and longitudinal resolution. Cross-well seismic ${ }^{[1]}$ has the advantages of high frequency and small bin. It can directly find out the cross-well reservoir's characteristics such as geometry, lateral distribution, connectivity, leading edge, property distribution, etc. Cross-well seismic can widen the bandwidth of the surface seismic signal, improve the resolution and identify the mini-structure. The most common cross-well seismic imaging method is based on VSP-CDP stack imaging ${ }^{[2,3]}$ which belong to ray imaging technology. It builds the velocity field by tomography or logging acoustic velocity curve, and uses the ray tracing method to migrate the reflection wave field to the correct position. The traditional VSP-CDP stack imaging method ${ }^{[4,5]}$ cannot solve the problem of the underground structure imaging in lateral changed severely. Based on the VSP-CDP stack method, this paper firstly uses cross-well seismic Gaussian beam forward method to replace conventional ray tracing method, and uses the idea of inverse Gaussian beam stacking to image the cross-well seismic data. It can effectively solve the problem of cross-well wave field imaging in complex geological structure.

\section{METHOD}

Cross-well seismic inverse Gaussian beam stack imaging includes two parts: the Gaussian beam forward modeling and inverse Gaussian beam stack. Cross-well seismic Gaussian beam forward modeling can provide accurate velocity information, reflection point coordinates, travel time and energy contribution of each ray to the receiver. Due to the energy of the receiver is the Gaussian weighted of surrounding effective rays, so the energy of each reflection point derive from the contributed receiver's inverse Gaussian decomposition when stack imaging. We choose a bin size to stack for obtaining morphological characteristics of subsurface structure after getting the energy of each reflection point.

\section{A. Gaussian beam forward modeling}

In order to overcome the certain limitations of ray trace method and wave equation method, Cerveny and others have developed a method which combined the wave equation and the ray theory, which was defined as Gaussian beam method ${ }^{[6-}$ ${ }^{9]}$.Gaussian beam forward modeling is based on the integral stake of Gaussian beam to obtain receiver wave field, which belongs to the ray tracing method and is the summarize and promotion of ray theory. Comparing with conventional ray methods, Gaussian beam forward modeling considers dynamic characteristics of the seismic wave. Gaussian beam forward modeling not only determine the wave ray path and calculate the wave propagation time, but also determine the seismic wave amplitude, waveform, particle vibration direction etc. It has wider range of application.

The Gaussian beam kinematics tracing is to calculate the ray path and the travel time transmitted from source to receive well. The travel time of seismic wave is calculated based on the eikonal equation at any point, such as formula 1 :

$$
\frac{\partial^{2} \tau}{\partial x^{2}}+\frac{\partial^{2} \tau}{\partial y^{2}}+\frac{\partial^{2} \tau}{\partial z^{2}}=\frac{1}{v^{2}}
$$

In formula $1, \tau$ is the seismic wave travel time and second is its unit. $\mathrm{v}$ is the velocity and its unit is $\mathrm{m} / \mathrm{s}$. 
$\mathrm{S}$ is the ray arc length along the direction of seismic wave transmitting(A positive engagement along the ray propagation direction). Because the ray and wave front $\tau(x, y, z)$ are orthogonal, The formula 2 can be gotten by the eikonal equation:

$$
\frac{d \tau}{d s}=\frac{1}{v}, d \tau=\frac{d s}{d v}
$$

$\mathrm{d} \tau / \mathrm{ds}$ is the directional derivative of $\tau$ along the direction of ray.

Integral $\tau$ along the arc s:

$$
\tau(s)=\tau\left(s_{0}\right)+\int_{s_{0}}^{s} \frac{d s}{v}
$$

If the initial value of $s_{0}$ point is given, then the travel time of s point can be calculated. The formula 3 is to calculate the seismic wave travel time.

Gaussian beam is the high frequency approximate solution of wave equation along the ray and it depends on the central ray. Through kinematics tracking, there will generate a center ray, then we do Gaussian beam dynamics tracking. The energy of receiver comes from all around the radiation energy Gaussian weighted. As shown in Figure 1, when the distance D between the adjacent ray and ray with the center is less than effective half width $L$ of receiver point $R$, the energy of the receiver is effective for all its Gaussian weighted superposition of radiation energy. Gaussian beam dynamic tracing rays is to obtain the dynamic parameters $\mathrm{P}$ and $\mathrm{Q}$. P is the component of slowness vector which is perpendicular to the direction of central ray, Q is the distance between the adjacent ray and the central ray. When we calculate the dynamic ray tracing equations, the initial value of $\mathrm{P}$ and $\mathrm{Q}$ should be given. Choosing the appropriate initial value in order to ensure that the Gaussian beam does not exist the singularity area of the wave field.

Hill had given the formula 4 about the initial value in $2001^{[10]}$.

$$
\left\{\begin{array}{c}
P_{0}=\left[\begin{array}{cc}
i / v_{0} & 0 \\
0 & i / v_{0}
\end{array}\right] \\
Q_{0}=\left[\begin{array}{cc}
4 \pi^{2} v_{0} / \omega & 0 \\
0 & 4 \pi^{2} v_{0} / \omega
\end{array}\right]
\end{array}\right.
$$

In formula $4, \mathrm{v} 0$ is the layer velocity in source, $\omega$ is radian frequency.

The values of $\mathrm{P}$ and $\mathrm{Q}$ in geophone are gotten shown in formula 5 through the initial conditions and the linear boundary conditions that the interface curvature is zero.

$$
\left\{\begin{array}{c}
\frac{\partial Q}{\partial s}=v P \\
\frac{\partial P}{\partial s}=-\frac{1}{v^{2}} \frac{\partial^{2} v}{\partial n^{2}} Q
\end{array}\right.
$$

In formula $5, \mathrm{~s}$ is the distance of seismic wave propagation, $\mathrm{n}$ is the left direction vector which is perpendicular to the beam propagation direction.

The wave field energy that seismic wave transmitted $\mathrm{s}$ meters in $2 \mathrm{D}$ can be shown in formula 6 :

$$
\begin{aligned}
& u(s, n, \omega)=\sqrt{\frac{v(s)}{Q(s)}} \exp (i \omega \tau(s) \\
& \left.+\frac{1}{2} \frac{P(s)}{Q(s)} n^{2}\right)
\end{aligned}
$$

The Gaussian beam expression is shown in formula 7, it is gotten by separating the real part and imaginary part of $\mathrm{P}(\mathrm{s})$ and $\mathrm{Q}(\mathrm{s})$ :

$$
\begin{aligned}
& u(s, n, \omega)=\sqrt{\frac{v(s)}{Q(s)}} \exp (i \omega \tau(s) \\
& +\frac{i w}{2 v(s)} K(s) n^{2}-\frac{n^{2}}{L^{2}(s)}
\end{aligned}
$$

In formula $7, \tau(\mathrm{s})$ represents the travel time of central \[ K(s)=v(s) \operatorname{Re}\left(\frac{P(s)}{Q(s)}\right) \text {, and it is the wave-front curvature of } \]
ray.
\[ L(s)=\left[\frac{\omega}{2} \operatorname{lm}\left(\frac{P(s)}{Q(s)}\right)\right]^{-\frac{1}{2}} \text {, and it is the effective } \]
Gaussian beam. half width of Gaussian beam.

\section{B. Stack imaging of inverse Gaussian beam}

As shown in Fig. 2, the energy of receiver point $\mathrm{R}$ is the Gaussian weighted of three rays energy form source. The energy of receive point $\mathrm{R}$ will be allocated to reflection points which contributed to the geophone in the idea of inverse Gaussian beam when imaging. The common reflection point (CRP) gathers are formed by stacking in a certain bin size in lateral. Through calculating the zero offset time between the adjacent layers, the reflection field record will be migrated on its corresponding reflection point in vertical. One trace of CRP gathers records will be generated in vertical using this method. Stack bin size is chosen in order to make no vacancy on stacked section, and then the CRP gathers will be stack to form the final stack section ${ }^{[11]}$.

\section{EXAMPLES}

\section{A. Model Test}

In order to test the adaptability and accuracy of cross-well seismic inverse Gaussian beam stacking imaging method, the geological model is established which contains a reverse fault, a normal fault and horizontal layer as shown in Fig.3. The layer velocities are $\mathrm{v} 1=2300 \mathrm{~m} / \mathrm{s}, \mathrm{v} 2=2500 \mathrm{~m} / \mathrm{s}, \mathrm{v} 3=2700 \mathrm{~m} / \mathrm{s}, \mathrm{v} 4$ $=3000 \mathrm{~m} / \mathrm{s}$, respectively. Table 1 is the cross-well seismic field geometry system parameters, Fig.4 is the reflection wave field recorded of cross-well seismic Gaussian beam forward( $\mathrm{P}$ reflection wave and $\mathrm{S}$ reflection wave). The stack imaging 
result is shown in Fig.5, it's processed by the cross-well seismic inverse Gaussian beam method and choosing the stack bin size as $10 \mathrm{~m}$. As shown in imaging result, it is consistent with subsurface geological structure and it can effectively reflect the real characteristics of subsurface complex structures. The result will provide the basis for further data processing.

\section{B. Actual Data Test}

In order to verify the application effect of cross-well seismic inverse Gaussian beam imaging method, we applied this method in the study of cross-well seismic reflection wave imaging in $\mathrm{K}$ area. According to the results of the study area ground seismic interpretation as shown in Fig.6, the initial geologic model is established as shown in Fig. 7. The initial velocity model is established through the logging acoustic curve characteristics which are shown in figure 8. Comparing the original record after wave field separation with forward reflection wave field record which generated by field crosswell seismic acquisition geometry system, adjusting the velocity model in order that the wave fields are identical as shown in Fig.9. The stack imaging section is obtained by using the final velocity model (Fig. 10) as the velocity field of inverse Gaussian, as shown in figure 11. Judging from the stack imaging result, the wave group relationships of target layers are clear, the signal noise ratio (SNR) is high, and the continuity of seismic event is better. Spectrum analysis is carried out on the section, the dominant frequency of crosswell seismic inverse Gaussian beam stack imaging section is $280 \mathrm{~Hz}$ in time domain, the bandwidth can reach $400 \mathrm{~Hz}$.

\section{CONCLUSION}

The cross-well seismic inverse Gaussian beam stack imaging method is applied on the theoretical model and actual seismic data, the results show that this method has higher resolution and can effectively solve the blind area problems which caused by subsurface complex geologic structure. The cross-well seismic inverse Gaussian beam stack imaging method has the characteristics of imaging accurately, higher computational efficiency, it can solve the problem of complex structure imaging and has important significance and broad application prospects in cross-well seismic data processing.

\section{A. Figures and Tables}

Table1 Geometry Parameters

\begin{tabular}{|c|c|c|c|c|}
\hline $\begin{array}{c}\text { Obser- } \\
\text { vation }\end{array}$ & $\begin{array}{c}\text { Shot } \\
\text { Number }\end{array}$ & $\begin{array}{c}\text { Trace } \\
\text { Number }\end{array}$ & $\begin{array}{c}\text { Sample } \\
\text { Interval(ms) }\end{array}$ & $\begin{array}{c}\text { Sampling } \\
\text { Point }\end{array}$ \\
\hline $\begin{array}{c}\text { Cross-well } \\
\text { Seismic }\end{array}$ & 8 & 120 & 1 & 3000 \\
\hline $\begin{array}{c}\text { Obser- } \\
\text { vation }\end{array}$ & $\begin{array}{c}\text { Shot } \\
\text { Interval } \\
\text { (m) }\end{array}$ & $\begin{array}{c}\text { Trace } \\
\text { Interval } \\
\text { (m) }\end{array}$ & $\begin{array}{c}\text { Shot } \\
\text { Range } \\
\text { (m) }\end{array}$ & $\begin{array}{c}\text { Receiver } \\
\text { Range } \\
\text { (m) }\end{array}$ \\
\hline $\begin{array}{c}\text { Cross-well } \\
\text { Seismic }\end{array}$ & 300 & 20 & $300-2400$ & $10-2390$ \\
\hline
\end{tabular}

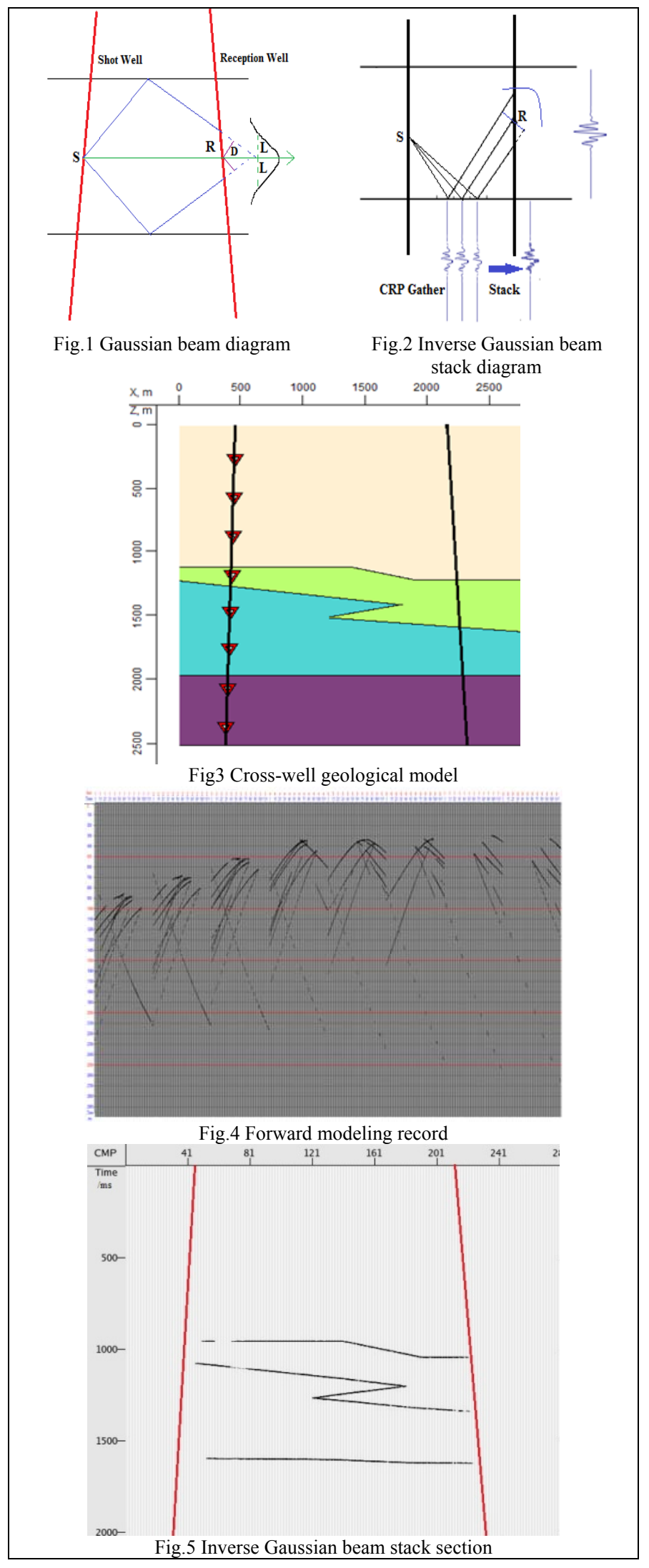




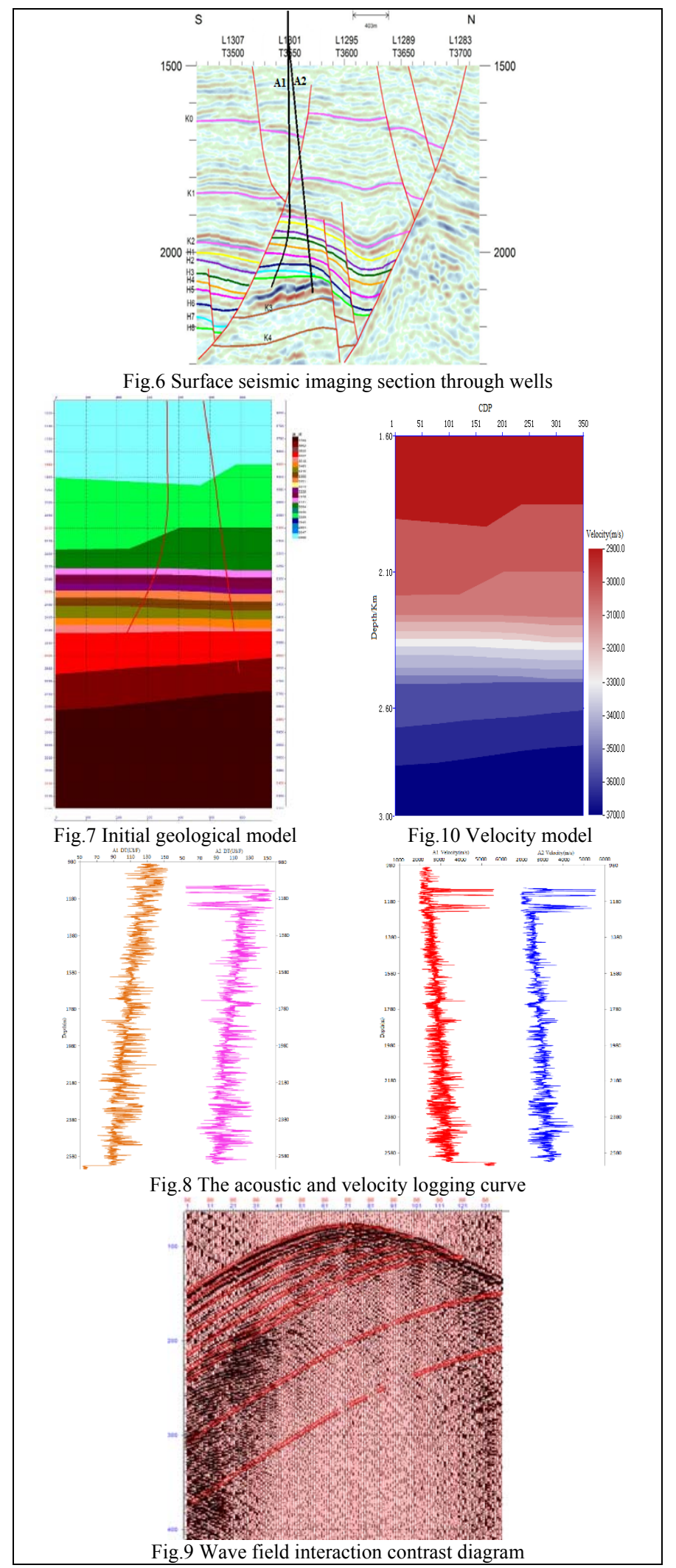

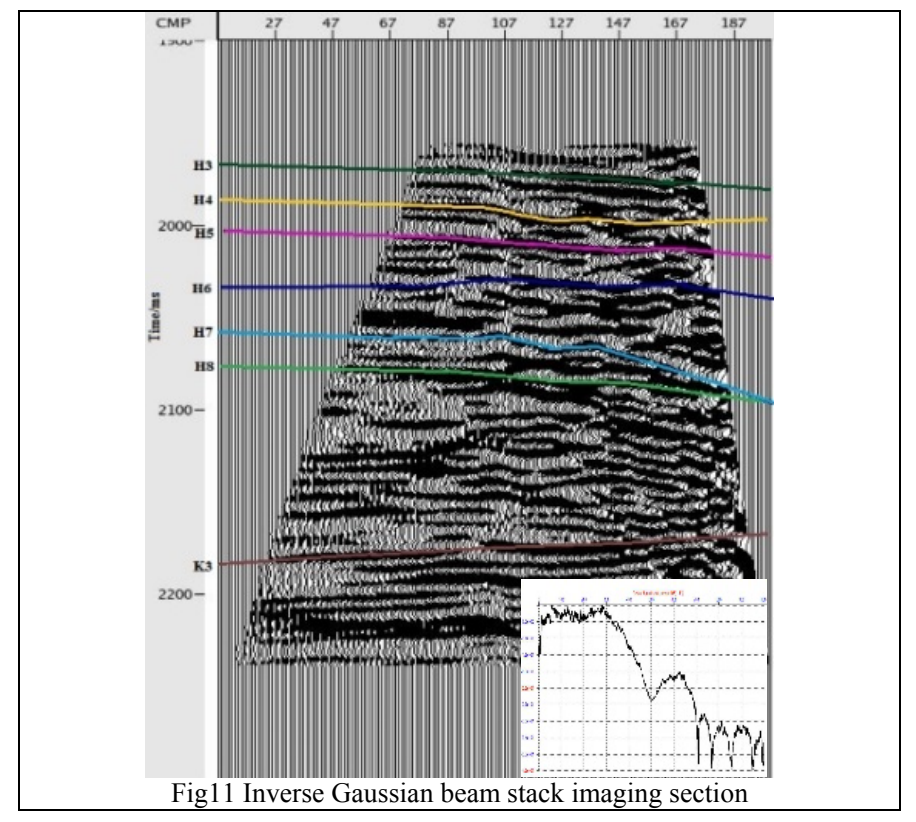

\section{REFERENCES}

[1] He Xing-hua. Cross-well Seismic[M]. Bei Jing: Petroleum Industry Press Inc. 2008

[2] K. Dautenhahn Wyatt. Synthetic vertical seismic profile[J]. GEOPHYSICS, 1981. 46(6), 880-891.

[3] Lazaratos, S.K., Reter, J.W., Harris, J.M., and Schaack, V.M.. HighResolution Imaging with Cross-Well Reflection Data:paper BG4.4 presented at the 1991 Annual Meeting of SEG, Hoston. 1991. Nov. 1014.

[4] Mark A. Van Schaack and Jesse C. Costa . XSP - CDP mapping in complex media without ray-tracing. SEG Technical Program Expanded Abstracts 1996: 1996.pp. 1890-1893.

[5] Yang Fei-long, Liu Dong-ming, Qin Min-jun, Su Zhi-dong. Interactive stack imaging method of VSP common reflection point in deviated well[J]. Computing Techniques for Geophysical and Geochemical Exploration. 2015. 37(6):728-734.

[6] V.Cerveny. Synthetic body wave seismograms for laterally varying layered structures by the Gaussian beam method[J].Geophysical Journal of the Royal Astronomical Society. 1983.73:389-426.

[7] V. Cerveny. Gaussian beam synthetic seismograms [J]. J Geophys. 1985.58:44-72.

[8] Hill,N.R. Gaussian beam migration[J]. Geophysics. 1990.55 (11) : 1416-1428.

[9] Yang Fei-long, Sun Yuan, Li Xu-xuan, et al. Deviated hole VSP forward method based on the Gaussian beam[J]. Progress in Geophysics(in Chinese), 2014.29(6):2791-2799.

[10] Hill,N.R. Prestack Gaussian beam depth migration[J].Geophysics. 2001.66(4):1240-1250.

[11] Yang Fei-long, Sun Yuan, Lu Jing. et al. The Gaussian beam stack imaging method of VSP[J]. Geophysical and Geochemical Exploration. 2015.39(3):627-632. 